\title{
MR Continuously Variable Transmission Driven by SMA for Centrifugal Fan in Nuclear Power Plant
}

\author{
Jianzuo Ma, ${ }^{1}$ Hongyu Shu, ${ }^{1}$ and Jin Huang ${ }^{2,3}$ \\ ${ }^{1}$ College of Mechanical Engineering, Chongqing University, Chongqing 400044, China \\ ${ }^{2}$ Chongqing Automobile College, Chongqing University of Technology, Chongqing 400054, China \\ ${ }^{3}$ The Key Laboratory of Manufacture and Test Techniques for Automobile Parts, \\ Chongqing University of Technology, Chongqing 400054, China \\ Correspondence should be addressed to Jianzuo Ma, mjzcqu@163.com
}

Received 15 June 2012; Revised 3 September 2012; Accepted 8 September 2012

Academic Editor: Yan Yang

Copyright ( $\odot 2012$ Jianzuo Ma et al. This is an open access article distributed under the Creative Commons Attribution License, which permits unrestricted use, distribution, and reproduction in any medium, provided the original work is properly cited.

The running efficiency of centrifugal fan affects the economical efficiency of the ventilation system. In this paper, we proposed a continuously variable transmission system based on magnetorheological fluid and shape memory alloy for improving the operating efficiency of the centrifugal fan. The equation of transmission torque developed by magnetorheological fluid is derived to compute the torque transmission ability in the continuously variable transmission system. A shape memory alloy spring actuator is designed to control the electric current in coil assembly. The results indicate that the change of temperatures has a tremendous influence on the electric current in coil assembly, the transmission torque of the continuously variable transmission system changes rapidly according to the temperatures acting on shape memory alloy spring actuator, and the output angular velocity of the centrifugal fan can be adjusted continuously.

\section{Introduction}

As an important component of ventilation system in nuclear power plant, the centrifugal fan plays an important role to ensure that the ventilation system is running reliably. The running efficiency of centrifugal fan affects the economical efficiency of the ventilation system. In a ventilation system, the flow rate or pressure should be timely changed according to the working conditions, so centrifugal fan should correspondingly adjust its velocity for improving the operating efficiency of the centrifugal fan. In order to solve the problem of traditional fan-driver which cannot export consecutive velocity, a magnetorheological (MR) continuously variable transmission system which is driven by shape memory alloy (SMA) is provided in this paper.

MR fluids and SMAs are known as smart materials for their properties can change rapidly on different external conditions. The yield stress of MR fluids changes rapidly and reversibly when an external magnetic field is applied [1-3].
MR fluids are useful for the efficient control of the torques and forces transmission; they can be used in clutches [4-8], brakes $[9,10]$, shock absorbers $[11,12]$, valves [13], and so on. SMAs may undergo mechanical shape changes at relatively low temperatures and retain them until heated, then coming back to the initial shape $[14,15]$. This makes SMAs unique compared to other smart materials that can be used for actuator applications [16-18].

An MR continuously variable transmission system transmits torque by the shear stress of the MR fluids from the driving shaft to driven shell. The MR continuously variable transmission system has the property that its transmitting torque changes quickly in response to an external magnetic field. Huang et al. [19] proposed the possibility of application of the MR fluids to variable speed transmission. Jiang et al. [20] gave a new type of self-pressurized structure of magnetorheological fluids continuously variable transmission (MRFCVT) with V-shape working gap. Ma et al. [21] derived the necessary working gap and the volume of MR fluid for 
the MR fan clutch based on MR fluid properties, the desired control torque ratio, the angular velocity, and load torque of the clutch.

In this paper, Herschel-Bulkley model is used to describe the constitutive characteristics of MR fluids subject to an applied magnetic field. The operational model of the continuously variable transmission system is established to derive the formula for the torque transmitted by MR fluids. A sliding mode SMA actuator is proposed to modify the magnetic field acting on working gap under thermal effect. The properties of the MR continuously variable transmission system are studied in detail to provide an effective approach for improving the operating efficiency of the centrifugal fan.

\section{Operational Principle}

The MR continuously variable transmission system relies on MR fluid as a transmission medium to transmit torque. The operational principle of the MR continuously variable transmission system which is driven by SMA is shown in Figure 1. Transmission shaft and driving disc are initiative members, and shell is a driven one. The initiative members rotate at definite velocity, $\omega_{1}$. The MR fluid fills the working gap between the driving disc and shell. The shell is joined to centrifugal fan. In the absence of magnetic field, MR fluid keeps flowing, so its transmission torque is only a very small viscous torque. However, a magnetic flux path is formed when electric current is put through the excitation coil. As a result, the magnetic particles in MR fluid are gathered to form chain-like structures, in the direction of the magnetic flux path. These chain-like structures restrict the motion of the MR fluid, thereby increasing the shear stress of the fluid. When the shear stress is large enough, initiative and driven members can finish a synchronous rotation.

Yield strength of MR fluid is a function of magnetic field strength [1], thus by changing magnetic field strength, the shear stress of MR fluid can be adjusted. So the transmitted torque of the MR continuously variable transmission system and the output rotate speed of driven shell can be modulated by varying the current in coil. The current in coil can be modulated by a sliding mode SMA actuator which alters the sliding distance according to the temperature acting on SMA spring, shown in Figure 2. The SMA helical spring works against a conventional steel spring (referred here as the "biasing" spring). At low temperatures, the steel spring is able to completely deflect the SMA spring to its compressed length. When increasing the temperature of the SMA spring, it expands, compressing the steel spring and moving the push rod.

\section{Properties of SMA and MR Fluid}

The most commonly used SMA elements for actuators are helical springs, for this form produces a large displacement. The force that a spring of any material produces at a given deflection depends linearly on the shear modulus of the material. SMAs exhibit a large temperature dependence on

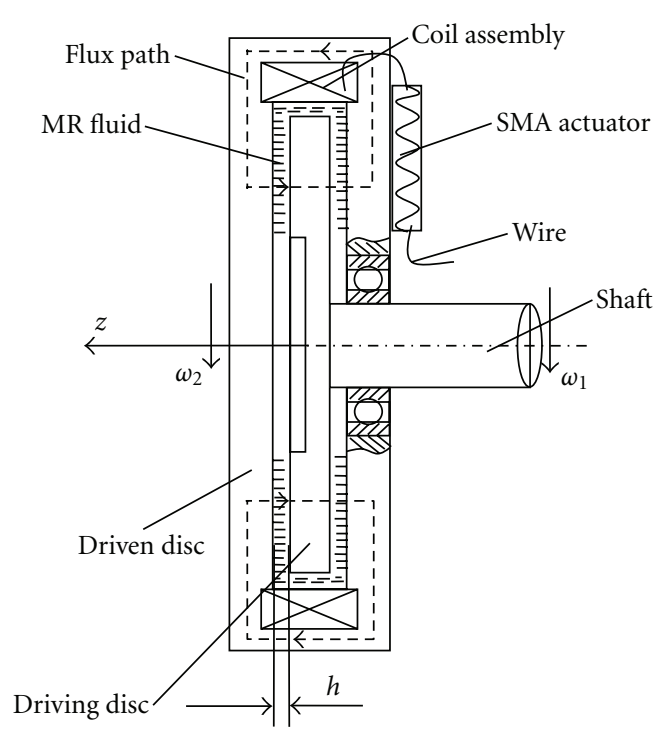

FIGURE 1: Operational principle of the MR continuously variable transmission system.

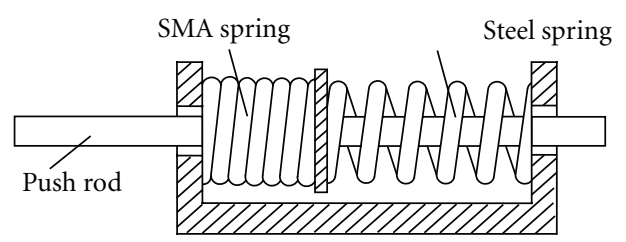

FIGURE 2: Operational principle of the SMA actuator.

the material shear modulus. The relationship between shear modulus and temperature for SMAs is given by

$$
G= \begin{cases}G_{M} & \text { when } T<M_{f}, T<A_{s} \\ G(T) & \text { when } M_{f} \leq T \leq A_{f} \\ G_{A} & \text { when } T>A_{f}, T>M_{s}\end{cases}
$$

where $G$ is the shear modulus of SMA, $T$ is temperature, $M_{s}, M_{f}, A_{s}$, and $A_{f}$ are the start and finish transformation temperatures of martensite and austenite, respectively, $G_{M}$ and $G_{A}$ are the shear modulus of martensite and austenite, respectively. When $M_{f} \leq T \leq A_{f}$, in absence of stress, shear modulus of SMA can be expressed approximately as:

$$
G(T)=G_{M}+\frac{G_{A}-G_{M}}{2}\left[1+\sin \phi\left(T-T_{m}\right)\right] .
$$

In the process of heating, $T_{m}=\left(A_{s}+A_{f}\right) / 2, \phi=\pi /\left(A_{f}-\right.$ $\left.A_{s}\right)$; in the process of cooling, $T_{m}=\left(M_{s}+M_{f}\right) / 2, \phi=$ $\pi /\left(M_{s}-M_{f}\right)$.

MR fluids exhibit a controllable yield stress-like behavior in shear, whereby the application of a magnetic field transverse to the flow creates a resistance to flow which increases with an increasing magnetic field. To accommodate 
the shearing thinning observed in MR fluids, the HerschelBulkley model [22] can be used to describe the flow behavior of MR fluid:

$$
\begin{gathered}
\tau_{2}=\tau_{y}(H)+K\left|\dot{\gamma}_{2}\right|^{m} \operatorname{sgn}\left(\dot{\gamma}_{2}\right) \quad \tau_{2} \geq \tau_{y}(H) \\
\dot{\gamma}_{2}=0 \quad \tau_{2}<\tau_{y}(H),
\end{gathered}
$$

where $\tau_{2}$ is the total shear stress of MR fluid, $\tau_{y}(H)$ is the yield strength caused by the applied magnetic field, $\dot{\gamma}_{2}$ is the shear rate of MR fluid, and $m, K$ are constants. In the Herschel-Bulkley model, the constants $m, K$ and the function $\tau_{y}(H)$ are empirically determined from experiments.

\section{Analysis of SMA Spring Actuator}

The scheme of the proposed actuator with aSMA spring and conventional steel against-spring is illustrated in Figure 2, where at low temperature the SMA spring will be compressed and when heated will extend with a pushing actuation.

The expression for shear stress in aSMA spring is described as

$$
\tau_{1}=\kappa \frac{8 F D}{\pi d^{3}}=\kappa \frac{8 F C}{\pi d^{2}} .
$$

Here, the axial load is $F, D$ is the average diameter of the spring, $d$ represents the wire diameter, $C$ is the spring index, $C=D / d$, and $\kappa$ is known as the Wahl correction factor applied:

$$
\kappa=\frac{4 C-1}{4 C-4}+\frac{0.615}{C} .
$$

The relationship between compressed length $\delta$ and shear strain $\gamma_{1}$ for SMA spring is given by

$$
\delta=\frac{n \pi D^{2}}{d} \gamma_{1}
$$

where $n$ is the number of turns in the spring.

The wire diameter for the actuator can be obtained from (4) for acceptable values of $C$ ranging from 3 to 12:

$$
d=\sqrt{\kappa \frac{8 F C}{\pi \tau_{1}}} .
$$
(6):

The number of turns in the spring can be obtained from

$$
n=\frac{\Delta \delta d}{\pi \Delta \gamma_{1} D^{2}},
$$

where $\Delta \delta$ represents the stroke of the actuator, and $\Delta \gamma_{1}$ is the strain difference at high and low temperatures:

$$
\Delta \gamma_{1}=\gamma_{L}-\gamma_{H}
$$

For SMA spring actuator in Figure 2, the axial load $F$ has the relationship with the compressed length of SMA spring $\delta$ as below:

$$
\begin{gathered}
\frac{F(T)}{\delta(T) G(T)}=\frac{F_{L}}{\delta_{L} G_{L}}, \\
F(T)=F_{L}+\frac{F_{H}-F_{L}}{\Delta \delta} S(T),
\end{gathered}
$$

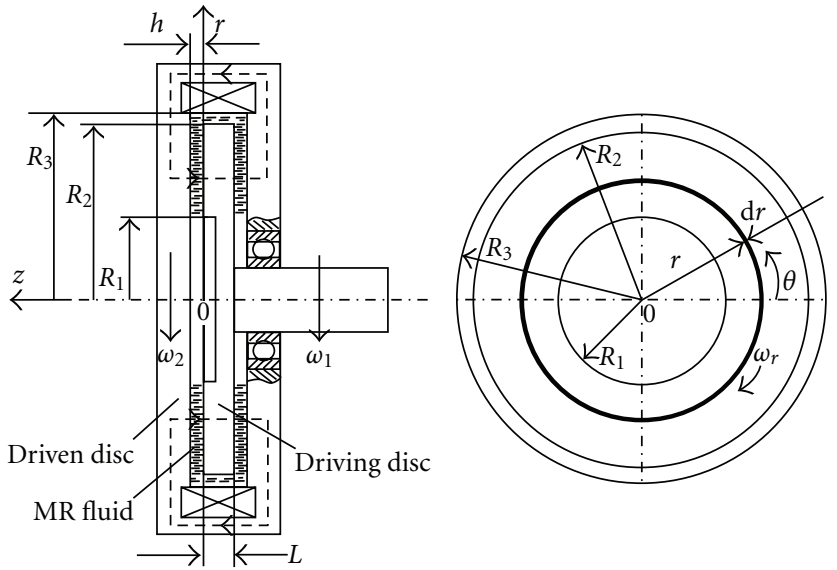

FIGURE 3: Circular flow mode of MR fluid between the two paralleled discs.

where $F(T), \delta(T)$, and $G(T)$ are the axial load, compressed length, and shear modulus of SMA spring at temperature $T$, respectively, $F_{L}, \delta_{L}$, and $G_{L}$ are the axial load, compressed length, and shear modulus of SMA spring at low temperature, respectively, $F_{H}$ is the axial load at high temperature, and $S(T)$ is the output displacement of SMA spring actuator:

$$
S(T)=\delta_{L}-\delta(T) .
$$

The output displacement of SMA spring actuator can be obtained from (1), (2), (6), (10), and (11):

$$
S(T)=\frac{\left(G(T)-G_{L}\right) \Delta \delta F_{L} \gamma_{L}}{\left(d / n \pi D^{2}\right) G(T) \Delta \delta F_{L}+\left(F_{H}-F_{L}\right) G_{L} \gamma_{L}} .
$$

\section{Analysis of Transmission Torque}

Figure 3 shows the flow behavior of MR fluid in the working gap between driving disc and shell. In order to determine the fluid flow between driving disc and shell, the following assumptions are given: the fluid is incompressible. There is no flow in radial direction and axial direction, but only tangential flow. The flow velocity of MR fluid is a function of radius. The pressure in the thickness direction of MR fluid is constant. The strength of magnetic field in the gap of the activation region is well distributed.

The angular velocity of MR fluid in the working gap can be obtained as follows.

At the range of $0 \leq z \leq h, R_{1} \leq r \leq R_{2}$ :

$$
\omega_{r}=\omega_{1}+\frac{\left(\omega_{2}-\omega_{1}\right) z}{h} .
$$

At the range of $R_{2}<r \leq R_{3}$ :

$$
\omega_{r}=\frac{R_{3}^{2} R_{2}^{2}}{R_{3}^{2}-R_{2}^{2}}\left[\left(\frac{R_{3}^{2}-r^{2}}{R_{3}^{2} r^{2}}\right) \omega_{1}+\left(\frac{r^{2}-R_{2}^{2}}{r^{2} R_{2}^{2}}\right) \omega_{2}\right] .
$$



by:

The fluid shear strain rate in (3) may be approximated

$$
\dot{\gamma}_{2}= \begin{cases}-r \frac{\mathrm{d} \omega_{r}}{\mathrm{~d} z} & R_{1} \leq r \leq R_{2} \\ r \frac{\mathrm{d} \omega_{r}}{\mathrm{~d} r} & R_{2} \leq r \leq R_{3} .\end{cases}
$$

Taking a microunit at distance $r$ location in a circle, microshear torque of the unit imposed on disc is as follows:

$$
\mathrm{d} J=\mathrm{d} F \cdot r=\left(\tau_{2} \cdot \mathrm{d} S\right) \cdot r .
$$

The total transmission torque is:

$$
J=\int_{r} \mathrm{~d} J
$$

Apply the boundary conditions of the continuously variable transmission system: $\omega_{r}=\omega_{1}$, at $z=0$ and $R_{1} \leq r \leq$ $R_{2} ; \omega_{r}=\omega_{2}$, at $z=h$ and $R_{1} \leq r \leq R_{2} ; \omega_{r}=\omega_{1}$, at $r=$ $R_{2} ; \omega_{r}=\omega_{2}$, at $r=R_{3}$. The transmission torque could be achieved from (13a), (13b), (14), (15), and (16):

$$
\begin{aligned}
J= & \frac{2 \pi}{3}\left(R_{2}^{3}-R_{1}^{3}\right) \tau_{y}(H)+\frac{2 \pi K}{m+3}\left(R_{2}^{m+3}-R_{1}^{m+3}\right)\left(\frac{\omega_{1}-\omega_{2}}{h}\right)^{m} \\
& +K\left[4 \pi L \frac{\left(\omega_{1}-\omega_{2}\right) R_{2}^{2} R_{3}^{2}}{R_{3}^{2}-R_{2}^{2}}\right]^{m}\left[\frac{\pi L}{2}\left(R_{2}+R_{3}\right)^{2}\right]^{1-m} .
\end{aligned}
$$

\section{Computational Results and Discussions}

Figure 4 shows the relation, obtained from the experiment, between the dynamic yield stress and the magnetic field strength for a typical MR fluid. From the figure we can find that the dynamic yield stress is proportional to the square of the magnetic field strength. MR fluid exhibits dynamic yield stresses of $0 \sim 30 \mathrm{kPa}$ for the applied magnetic field strength of $0 \sim 175 \mathrm{kAmp} / \mathrm{m}$. The ultimate strength of MR fluid is limited by magnetic saturation. The result shows that, with the increase of the applied magnetic field strength, the dynamic yield stress goes up rapidly.

According to (12) and (2), the effect of temperature in output displacement of SMA spring actuator can be analyzed, show as in Figure 5. In this study, Ti-49.8 at. $\% \mathrm{Ni}$ SMA wire is used, its start and finish temperatures of the martensitic and austenitic phase transformation are $M_{s}=78^{\circ} \mathrm{C}, M_{f}=50^{\circ} \mathrm{C}, A_{s}=74^{\circ} \mathrm{C}$, and $A_{f}=95^{\circ} \mathrm{C}$, respectively. The shear moduli of martensite and austenite are $G_{M}=7.5 \mathrm{GPa}$ and $G_{A}=25 \mathrm{GPa}$, respectively. The axial loads of SMA spring at low and high temperatures are $F_{L}=15 \mathrm{~N}$ and $F_{H}=40 \mathrm{~N}$, respectively. The stroke of the actuator is $\Delta \delta=20 \mathrm{~mm}$. Assume that the low temperature shear strain is $\gamma_{L}=1.5 \%$ and the value of spring index is $C=7$. The wire diameter of SMA spring for the actuator which can be obtained from (7) is $d=1.7 \mathrm{~mm}$, the number of turns which can be obtained from (8) is $n=25$. As shown in Figure 5, the output displacement of SMA spring actuator increases with the increasing of temperature that can be controlled by temperature.

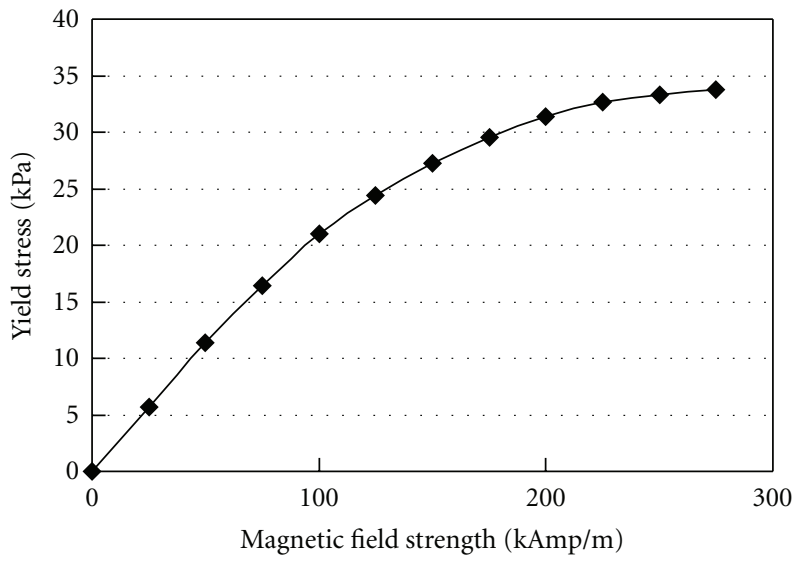

FIGURE 4: Yield stress versus magnetic field strength.

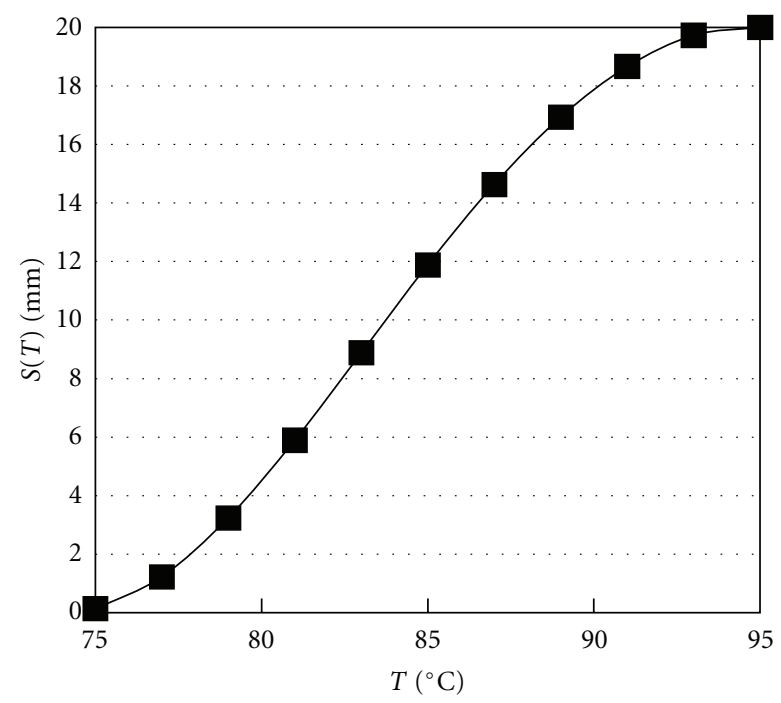

Figure 5: The output displacement versus temperature.

According to (17), the effect of magnetic field strength in transmission torque of the MR continuously variable transmission system is analyzed, as shown in Figure 6. The viscosity of the typical MR fluid is $0.042 \mathrm{~Pa} \cdot \mathrm{s}$. The transmission torque is mainly produced by yield stress of MRF and viscosity torque is very small, so it can be assumed that $m=1, K=0.042$ Pa.s in the Herschel-Bulkley model. Geometric parameters of the continuously variable transmission system are inner radius $R_{1}=60 \mathrm{~mm}$, outer radius $R_{2}=120 \mathrm{~mm}, R_{3}=122 \mathrm{~mm}$, working gap $h=$ $1 \mathrm{~mm}, L=4 \mathrm{~mm}$. The maximum input angular velocity is $\omega_{1 \max }=233 \mathrm{rad} / \mathrm{s}$. The transmission torques are $72.5 \mathrm{~N} \cdot \mathrm{m}$, $130.4 \mathrm{~N} \cdot \mathrm{m}, 173.1 \mathrm{~N} \cdot \mathrm{m}$, and $212.8 \mathrm{~N} \cdot \mathrm{m}$ at the magnetic field strength of $50 \mathrm{kAmp} / \mathrm{m}, 100 \mathrm{kAmp} / \mathrm{m}, 150 \mathrm{kAmp} / \mathrm{m}$, and $200 \mathrm{kAmp} / \mathrm{m}$, respectively. The results indicate that with the increase of magnetic field strength the transmission torque is increased.

The output angular velocity versus various temperatures is shown in Figure 7. It is assumed that the torque of lord 


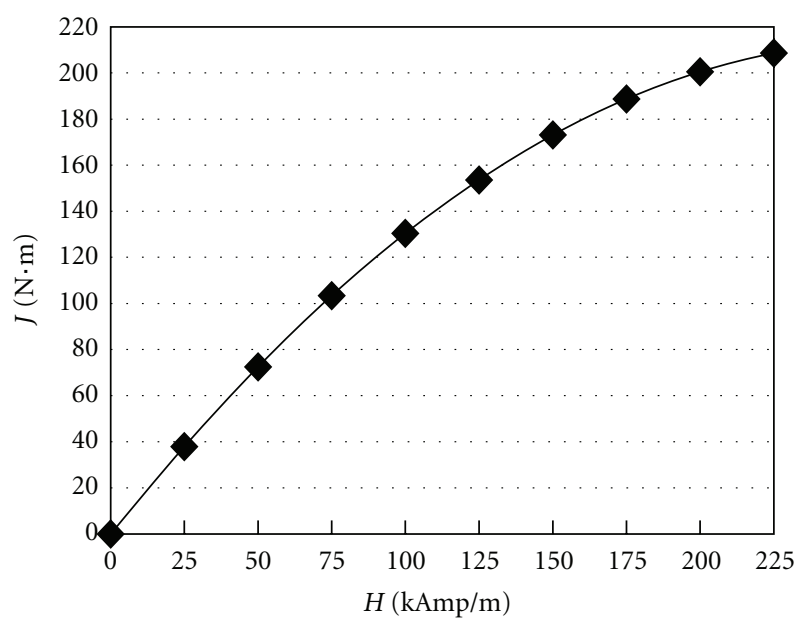

Figure 6: Transmission torque under different magnetic field strength.

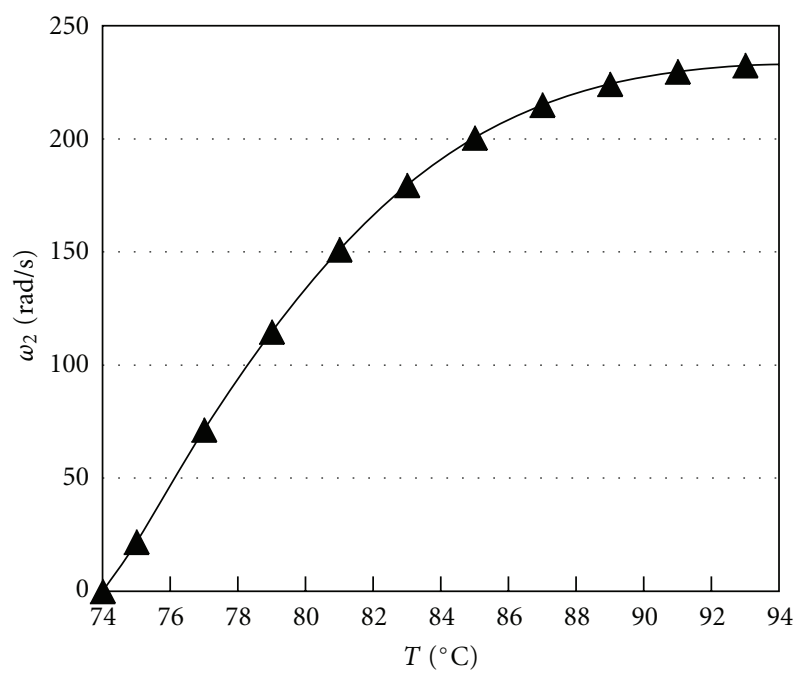

FIgURE 7: The output angular velocity versus temperature.

can be calculated from the empirical formula as $\left(J_{L}=5.5\right) \times$ $\left(10^{-5}\right) \times\left(30 \omega_{2} / \pi\right)^{2}$ approximately, the current in excitation coil increases by $0.1 \mathrm{~A}$ as push rod of SMA actuator goes ahead $1 \mathrm{~mm}$, and magnetic field strength produced by excitation coil increases $10 \mathrm{kAmp} / \mathrm{m}$. The result shows that with the increase of the temperature, the output angular velocity is increased.

\section{Conclusions}

The design method of an MR continuously variable transmission system driven by SMA is investigated theoretically in this paper. The equation of transmission torque developed by the MR fluid is derived. The transmission torque of the continuously variable transmission system under different magnetic field strength is analyzed. An SMA spring actuator is designed to control the transmission torque of the continuously variable transmission system. The output angular velocity of the transmission system versus temperatures is analyzed. With the increase of the applied magnetic field strength, the transmission torque of the continuously variable transmission system is increased. The output displacement of SMA spring actuator is controlled by temperature. The output angular velocity of the transmission system increases with the increasing of temperature acting on SMA actuator, rapidly and adaptively.

\section{Acknowledgments}

This work was supported by project 51175532 supported by the National Natural Science Foundation of China and key Project 2011BA4028 supported by Natural Science Foundation Project of CQ CSTC.

\section{References}

[1] X. Q. Peng, F. Shi, and Y. F. Dai, "Magnetorheological fluids modelling: without the no-slip boundary condition," International Journal of Materials and Product Technology, vol. 31, no. 1, pp. 27-35, 2008.

[2] J. Zhang, J. Q. Zhang, and J. F. Jia, "Characteristic analysis of magnetorheological fluid based on different carriers," Journal of Central South University of Technology, vol. 15, no. 1, pp. 252-255, 2008.

[3] M. S. Kim, Y. D. Liu, B. J. Park, C. Y. You, and H. J. Choi, "Carbonyl iron particles dispersed in a polymer solution and their rheological characteristics under applied magnetic field," Journal of Industrial and Engineering Chemistry, vol. 18, no. 2, pp. 664-667, 2012.

[4] A. S. Shafer and M. R. Kermani, "On the feasibility and suitability of MR fluid clutches in human-friendly manipulators," IEEE/ASME Transactions on Mechatronics, vol. 16, no. 6, pp. 1073-1082, 2011.

[5] T. Kikuchi, K. Otsuki, J. Furusho et al., "Erratum: development of a compact magnetorheological fluid clutch for humanfriendly actuator (Advanced Robotics (2010) 24 (14891502))," Advanced Robotics, vol. 25, no. 9-10, p. 1363, 2011.

[6] P. Kielan, P. Kowol, and Z. Pilch, "Conception of the electronic controlled magnetorheological clutch," Przeglad Elektrotechniczny, vol. 87, no. 3, pp. 93-95, 2011.

[7] A. L. Smith, J. C. Ulicny, and L. C. Kennedy, "Magnetorheological fluid fan drive for trucks," Journal of Intelligent Material Systems and Structures, vol. 18, no. 12, pp. 1131-1136, 2007.

[8] Z. Herold, D. Libl, and J. Deur, "Design and testing of an experimental magnetorheological fluid clutch," Strojarstvo, vol. 52, no. 6, pp. 601-614, 2010.

[9] J. Huang, J. Q. Zhang, Y. Yang, and Y. Q. Wei, "Analysis and design of a cylindrical magneto-rheological fluid brake," Journal of Materials Processing Technology, vol. 129, no. 1-3, pp. 559-562, 2002.

[10] A. Farjoud, N. Vahdati, and Y. F. Fah, "Mathematical model of drum-type MR brakes using herschel-bulkley shear model," Journal of Intelligent Material Systems and Structures, vol. 19, no. 5, pp. 565-572, 2008.

[11] A. Milecki and M. Hauke, "Application of magnetorheological fluid in industrial shock absorbers," Mechanical Systems and Signal Processing, vol. 28, pp. 528-541, 2012.

[12] E. Dragasius, V. Grigas, D. Mazeika, and A. Sulginas, "Evaluation of the resistance force of magnetorheological fluid 
damper," Journal of Vibroengineering, vol. 14, no. 1, pp. 1-6, 2012.

[13] J. Huang, J. M. He, and J. Q. Zhang, "Viscoplastic flow of the MR fluid in a cylindrical valve," Key Engineering Materials, vol. 274-276, no. 1, pp. 969-974, 2004.

[14] C. Yu, G. Z. Kang, D. Song, and Q. H. Kan, "Micromechanical constitutive model considering plasticity for super-elastic NiTi shape memory alloy," Computational Materials Science, vol. 56, pp. 1-5, 2012.

[15] S. Huang, M. Leary, T. Ataalla, K. Probst, and A. Subic, "Optimisation of Ni-Ti shape memory alloy response time by transient heat transfer analysis," Materials and Design, vol. 35, pp. 655-663, 2012.

[16] A. Hadi, A. Yousefi-Koma, M. Elahinia, M. M. Moghaddam, and A. Ghazavi, "A shape memory alloy spring-based actuator with stiffness and position controllability," Proceedings of the Institution of Mechanical Engineers Part I, vol. 225, no. 17, pp. 902-917, 2011.

[17] S. Langbein and A. Czechowicz, "Adaptive resetting of SMA actuators," Journal of Intelligent Material Systems and Structures, vol. 23, no. 2, pp. 127-134, 2012.

[18] T. Georges, V. Brailovski, and P. Terriault, "Characterization and design of antagonistic shape memory alloy actuators," Smart Materials and Structures, vol. 21, no. 3, Article ID 035010, 2012.

[19] J. Huang, G. H. Deng, Y. Q. Wei, and J. Q. Zhang, "Application of magnetorheological fluids to variable speed transmission," in Proceedings of the International Conference on Mechanical Transmissions (ICMT'01), pp. 296-298, April 2001.

[20] J. D. Jiang, X. C. Liang, and B. Zhang, "Research of selfpressurized MR continuously variable transmission," in Proceedings of the Smart Materials for Engineering and Biomedical Applications, pp. 386-390, 2004.

[21] J. Z. Ma, G. C. Wang, and D. Zuo, "Geometric analysis in an MR fan clutch," Advanced Materials Research, vol. 239-242, pp. 1731-1734, 2011.

[22] W. H. Herschel and R. Bulkley, "Consistency measurements of rubber-benzol solutions," Kolloid-Zeitschrift, vol. 39, no. 4, pp. 291-300, 1926. 

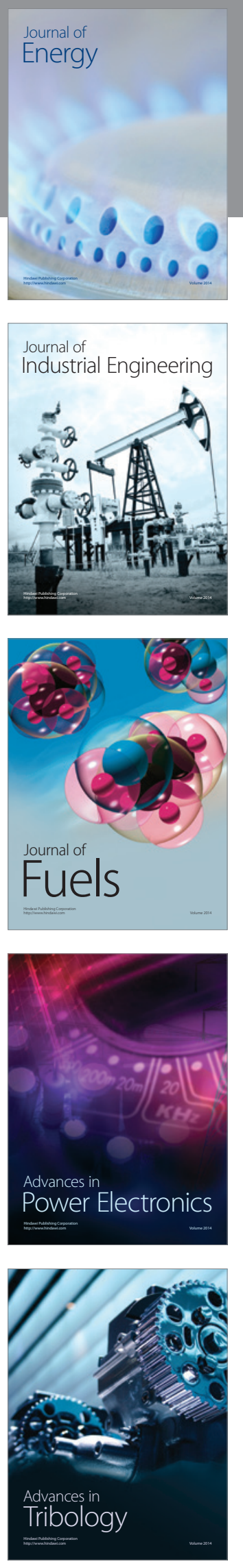
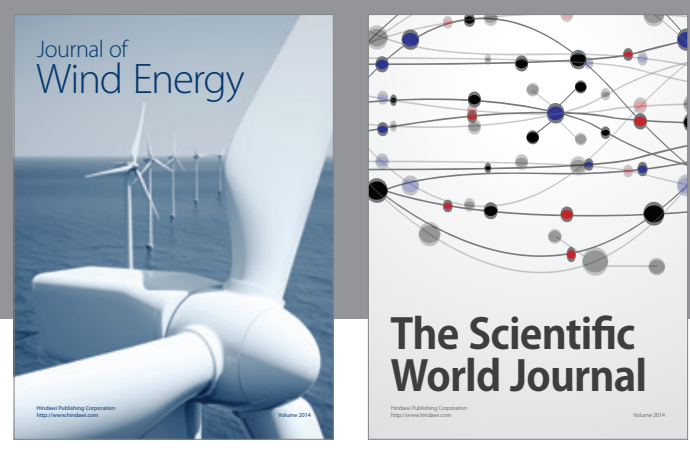

The Scientific World Journal

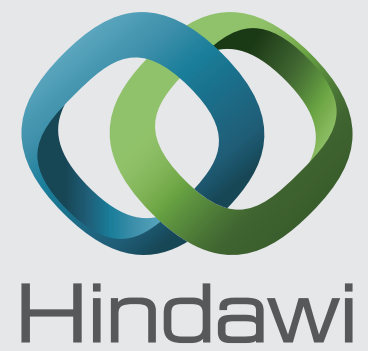

Submit your manuscripts at http://www.hindawi.com
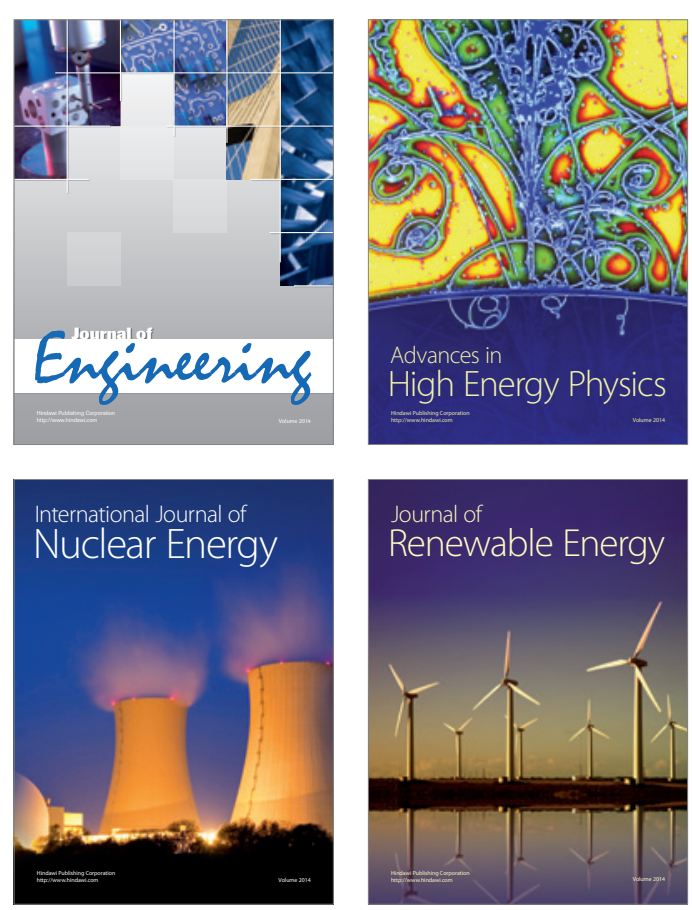

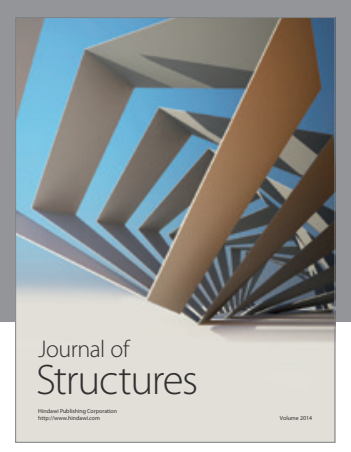

Rotating
Mechinery
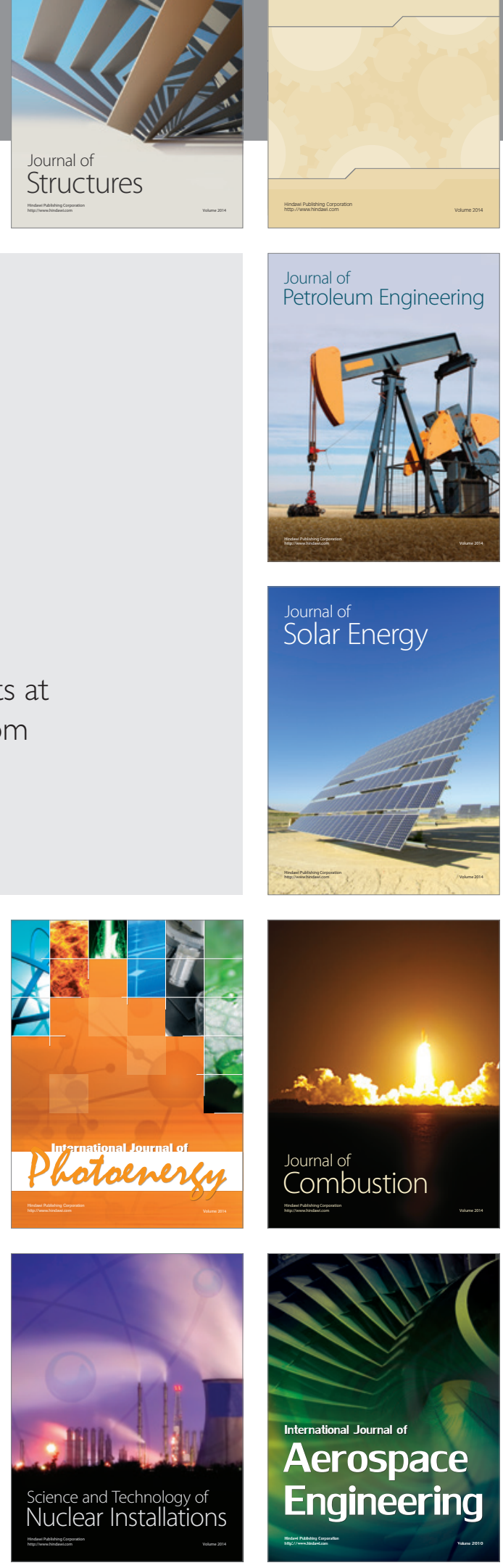